\title{
Ausblick auf das Ende des kolonialen Denkens
}

\author{
Edgar Forster
}

„The master's tools

will never dismantle the master's house.“

(Lorde, 1984)

Einen ,Ausblick auf das Ende des kolonialen Denkens‘ zu versuchen, ist ein imaginatives Unterfangen, denn der Abschied vom kolonialen Denken bedeutet das Ende der europäischen Moderne. Das ist die zentrale These des lateinamerikanischen kollektiven Projekts modernidad/colonialidad (vgl. Escobar, 2007). Studien zur kolonialen Differenz wie jene von Walter Mignolo (vgl. 2012) und das Werk von Michael Wimmer bilden die Grundlage für die folgenden Aufzeichnungen, die einen Übergang zu einem dekolonialen Denken und zu einer dekolonialen Praxis imaginieren. Sie haben ihren Ausgangspunkt in einem Unbehagen über die Figur und den Begriff des Anderen im wissenschaftlichen und philosophischen Diskurs. Ist die Erfindung des Anderen eine Demonstration des kolonialen Denkens par excellence, ein - weiterer - Akt der (Re-)Souveränisierung europäischer Vernunft? Welches Denken ist stark genug, um mit diesen Souveränisierungen zu brechen und das Denken der europäischen Moderne zu provinzialisieren (vgl. Chakrabarty, 2008)?

\section{1}

Die Dekonstruktion ist der radikalste Versuch, das Denken der europäischen Moderne ,von innen heraus' und mit seinen eigenen Mitteln zu ,provinzialisieren', das heißt, seinen Horizont, seinen Wirkungskreis und seine Grenzen zu bestimmen. Auf rigorose und virtuose Weise repräsentiert Wimmers Werk die Dekonstruktion in der Erziehungswissenschaft. Der Stil seines Werks ist bemerkenswert; er produziert eine doppelte Sprache. In Der Andere und die Sprache wird die Schreibpraxis der Dekonstruktion von einem anderen ,Schriftzug، überlagert oder durchquert, und man müsste dieser Art des Fragens, dem überstürzten Allessagen, dem Vorwegnehmen und dem nachträglichen Hinzufügen im gesamten Werk Wimmers Beachtung schenken. Der Stil der doppelten Sprache setzt seinen Autor zur Dekonstruktion in ein Verhältnis. Der Abstand zur Dekonstruktion begrenzt sie. Die Praxis des ,Sich-zur-Dekonstruktion-ins-Verhältnis-Setzen-und-sie-Begrenzen“ sollten uns nicht dazu veranlassen, hinter die Dekonstruktion zurückzugehen - Wimmer hat die Kritik, dass die Dekonstruktion zu weit gehe, immer zurückgewiesen - und auch nicht, dass man über sie hinausgehen und sie radikalisieren müsse. Jede Überschreitung ist eine dekonstruktive Praxis. Sich zur Dekonstruktion ins Verhältnis zu setzen, eröffnet die Möglichkeit, einen Ausgang und Übergang zu ,etwas anderem‘ zu finden. Obwohl sich die Dekonstruktion nicht erschöpft, kommt sie an eine Grenze. Das ist ihr Mangel und ihre Qualität.

\section{2}

Die Dekonstruktion ist eine Praxis, ein Eingriff, eine Setzung. Die Setzung hat immer einen Bezug zu einem Anderen, zu einer Andersheit. Im französischen Original steht für „Setzung“ das Wort position, und Derrida (1986, S. 177) verwendet viel Sorgfalt auf die Unmöglichkeit der „Setzung dieser Andersheit als solcher“. Die Andersheit des Anderen sei das, was in keinem Fall „gesetzt“ werden könne, wodurch jede Setzung durch sich selbst vereitelt werde. 
Die Unmöglichkeit der Setzung setzt eine Bewegung in Gang: „Kettenreaktionen“ (ebd., S. 86), Verschiebungen, „Abzug“, „Verpflanzung“, „Ausweitung“(ebd., S. 183).

Die Dekonstruktion bleibt der position verbunden, so wie différance dem Begriff différence verbunden bleibt. Wenn man nicht die endlose Verschiebung und Suspension der dekonstruktiven Praxis und damit die Andersheit des Anderen hervorhebt, sondern die Praxis des Eingriffs und der Positionierung, dann wird der locus of enunciation bedeutsam. Der Eingriff ist eine Praxis des Aussagens, und das Sagen ist ein Eingriff. Sie unterbricht das Gedachte und die Kritik. Der Eingriff (die Unterbrechung, der Einschnitt) markiert einen Ort; er begrenzt das Sagen. Das Prinzip des Aufschiebens und Verschiebens überlagert die Limitation dieser Praxis. In den späteren Schriften führt Derrida unablässig und immer detaillierter die ,Orte‘ ein, von denen aus er spricht, eingreift und die Dekonstruktion ins Werk setzt.

Die Dekonstruktion ist eine Praxis: poser, positionner, se positionner. Ihr Bezug zum locus of enunciation ist nicht arbiträr. Die Dekonstruktion ist nur von einem Ort aus möglich. Die Dekonstruktion ist eine Praxis, die vom locus of enunciation affiziert ist. Sie bestimmt räumliche und zeitliche Verhältnisse, Abstände und Relationen der Macht, des Affekts, des Begehrens. Aus diesem Grund ist die Dekonstruktion keine abstrakte Übung; sie kann keine Methode werden. Jede Form der Universalisierung ist ihr fremd. Sie begründet keine neue Souveränität des Denkens. Das erklärt die Stellung des Fragens in Wimmers Werk. Ohne Limitation keine Dekonstruktion.

\section{3}

Über einen Zeitraum von mehr als dreißig Jahren hat Michael Wimmer die europäische Moderne im pädagogischen Denken dekonstruiert, ihre Aporien und Paradoxien freigelegt, den Einsatz der Dekonstruktivismus in der Erziehungswissenschaft begründet und die Leistungsfähigkeit dekonstruktiver Einsätze demonstriert (vgl. z.B. Wimmer, 2006, S. 353382). Im Vorwort zu seinem ersten Buch Der Andere und die Sprache skizziert er den Problemhorizont seines Denkens:

„Fraglich ist, wie man einen Diskurs weiterführen kann, der zwar programmatisch die Humanisierung der Welt zum Ziel hatte, der aber weder den Rückfall in die Barbarei voraussehen, geschweige denn verhindern konnte, noch die Technisierung der Welt als Preis für deren Zivilisierung. Zweifel sind aufgekommen daran, daß die technisch instrumentalisierte Welt bloßes Mittel sein kann für die Zwecke des Menschen, daß der Fortschritt in den Wissenschaften und in der Naturbeherrschung, mithin die Modernisierung der Gesellschaft wirklich den Zielen einer Humanisierung dient“ (Wimmer, 1988, Vorwort, o.S.).

Jede Erwägung über die Moderne vergrößert das Misstrauen in ihre zentralen Absichten. Die transatlantische Kolonialgeschichte, ein europäisches Projekt, kontaminiert die Idee der Humanisierung, und die in der Dialektik der Aufklärung gewählte Perspektivierung der Barbarei als einen ,Rückfall ‘ wirkt an diesem Vergessen der europäischen Kolonialgeschichte mit. Fortschritt, Zivilisierung, Humanisierung - die Konzepte der Moderne repräsentieren eine epistemische Gewalt. Sie legitimieren die systematische Ausrottung und Ausbeutung von Menschen.

Welche Konsequenzen muss die Erziehungswissenschaft aus dem Umstand ziehen, dass das pädagogische Denken in dieser europäischen Moderne seine Wurzeln hat, aus ihr seine Legitimation bezieht und zu ihrer Reproduktion einen wichtigen Beitrag leistet? Wimmers Beschreibung legt folgende Interpretation nahe: Der Zusammenhang zwischen der europäischen Moderne und der Gewalt in seinen verschiedenen Formen ist systematisch. Barbarei ist ein konstitutives Element der europäischen Moderne, und Zivilisierungs- und 
Humanisierungspraktiken sind ein Teil dieses Problems. Wenn pädagogisches Denken das Resultat und die Antriebskraft dieser Moderne ist, wenn sie, kurz gesagt, diese Moderne ist, dann ist das pädagogische Denken in seiner Totalität affiziert, und nur ein epistemologischer Bruch verändert das Verhältnis zur Barbarei.

\section{4}

Michael Wimmers Denken zielt auf einen epistemologischen Bruch. Eine theoretische Inspiration dafür bildet das Berliner Projekt ,Historische Anthropologie‘, das Phänomene und Strukturen des Menschlichen nach dem Ende der Verbindlichkeit einer abstrakten anthropologischen Norm denkt (vgl. Wulf/Kamper, 2002). Die Historische Anthropologie bezieht die Geschichtlichkeit ihrer Perspektiven und Methoden auf die Geschichtlichkeit ihres Gegenstandes. Der Mensch verliert seine zentrale Stellung in der Produktion von Sinn. Im eben zitierten Vorwort zeigt Wimmer die Richtung seines Denkens an:

„Die These vom Ende des Menschen ist deshalb als Versuch zu verstehen, dieser Problematisierung, diesem Denken des Menschen und diesem Dilemma zu entkommen, um, wie Foucault schreibt, wieder philosophieren zu können. Dazu muß der Zusammenhang von Vernunft und Gewalt, von Humanwissenschaft und Machtdispositiven, von Identitätslogik und Ethik, wie er sich im Humanismus und im neuzeitlichen Prinzip der Subjektivität manifestiert, unterbrochen werden. Das heißt jedoch, daß weder das Problem des Anderen noch die Frage nach dem Subjekt hinfällig geworden sind, sondern daß es darum geht, ein anderes Verständnis des Menschen zu entwickeln, das ein anderes Verhältnis zum Anderen, zur Natur und zur Geschichte möglich macht. Deshalb ist es keineswegs eine Resignation oder das Eingeständnis, mit der Kritik zu weit gegangen zu sein, wenn heute wieder die Frage nach dem Menschen und dem Subjekt aufgenommen wird in der Diskussion um die Menschenrechte und um Probleme der Ethik und Ästhetik“ (Wimmer, 1988, o.S.).

„Ein anderes Verständnis des Menschen“ sowie „ein anderes Verhältnis zum Anderen, zur Natur und zur Geschichte“ verweisen auf einen epistemologischen Bruch, weil es nicht um eine Humanisierung des Verhältnisses zum Anderen, um einen aufgeklärten Blick auf die Geschichte oder um ein nachhaltiges Engagement für die Natur geht, sondern um ein anderes Denken dieser Verhältnisse und ihrer Elemente. Wenn der Begriff des Subjekts der Aufklärung verblasst und seine Stellung im Erkenntnisprozess einbüßt, dann verändert sich die Beziehung zum Anderen - er ist dem Subjekt so wenig entgegengesetzt wie die Natur der der Kultur -, und der Mensch verliert seine Dominanz in der Welt der Dinge und des Lebens.

\section{5}

Der Begriff „epistemologischer Bruch“ rekurriert auf Gaston Bachelard und die Tradition der Historischen Epistemologie, in der wissenschaftliches Wissen durch einen Bruch mit der Wirklichkeit, wie sie uns entgegentritt, entsteht. Naturwissenschaft zielt nicht darauf ab, dass das erkennende Subjekt die Gegenstände unverstellt abbildet. An die Stelle der Wahrheit der ,Repräsentation' tritt ein Erkenntnisprozess, in dem ,etwas' durch eine experimentale Anordnung ,realisiert“ wird. Historische Epistemologie fragt nach den „Bedingungen, die geschaffen wurden oder geschaffen werden müssen, um Gegenstände unter jeweils zu bestimmenden Umständen zu Gegenständen empirischen Wissens zu machen“ (Rheinberger, 2007, S. 12). Der „epistemologische Bruch“ tritt als Bruch zwischen einem Realismus der ersten Ordnung und einem der zweiten Ordnung auf. Der Realismus der zweiten Ordnung besteht aus ,Realisierungen“. Darunter versteht Rheinberger (ebd., S. 39) einen „Prozess der ,Verwirklichung‘ um das, was sein kann“ (ebd., S. 39). Realisierungen und Imaginationen 
sind systematisch aufeinander bezogen. Jede Realisierung produziert neue Imaginationen, die wiederum Realisierungen ermöglichen. Dieser Prozess ist kontingent, aber keineswegs beliebig; er hat einen Nexus zur Welt der Technik: „So wie im Innersten des experimentellen Verfahrens alles um Technophänomene kreist, so sind die Naturwissenschaften als Ganzes sozial nur aus dem Geiste der Technik zu verstehen und auf diese als das Feld ihrer Realisierung im gesellschaftlichen Maßstab bezogen“ (ebd., S. 44).

Die Historische Epistemologie ist keine Wissenschaftsphilosophie der Naturwissenschaften, auch wenn sie sich in diesem Feld entwickelt und daraus ihre Inspiration erhalten hat. Wie naturwissenschaftliche Experimente umfassen Forschungen in den Humanwissenschaften Praktiken - Schreiben, Lesen, Recherchieren, Dokumentieren sowie Quellen des Wissens und der Imagination, die man als Archiv bezeichnen kann. Humanwissenschaftliche Forschungen benötigen erstens ein Wissen über das Entziffern von Dokumenten oder, allgemein, über den Umgang mit Archiven und zweitens die Imagination und Kenntnis, Wissenselemente in eine Anordnung zu bringen, um daraus etwas Neues entstehen zu lassen. Bachelard (1988) beschreibt den wissenschaftlichen Fortschritt als Evolutionsprozess, der mit einem epistemologischen Bruch beginnt und sich auf der Ebene des Realismus zweiter Ordnung durch ,epistemische Brüche‘ entwickelt (eine Theorie löst eine andere $a b)$. Häufiger handelt es sich nicht um einen linearen Fortschritt, sondern um eine Dissemination (ein neues Paradigma verändert die Begriffe, Fragen und Denkrichtungen), ohne dass ein neues Paradigma das vorherige ersetzt. Der Widerstreit erzeugt andere Experimentalanordnungen. Sie realisieren andere Verkoppelungen oder Möglichkeiten. Die Geschichte der Humanwissenschaften ist eine Geschichte der produktiven Koppelungen. Sie entstehen durch eine andere Lektüre - Derridas Werk und die deleuzianische Kant-Lektüre sind dafür ein Beispiel - oder durch eine erfinderische Schreibpraxis - Deleuze und Guattari definieren das Erfinden von Begriffen als die Aufgabe der Philosophie - oder durch einen imaginativen Gebrauch des Archivs - Foucaults Archäologien bezeugen einen solchen Gebrauch - oder durch die Kreation von Archiven, die zuvor nicht als Archive gesehen worden sind, oder die Produktion von Archiven durch Aufzeichnungen oder ethnographische Erkundungen usw.

Das Archiv ist keine Fundgrube für Fakten über eine vergangene oder (noch) gegenwärtige Realität. Die Historische Epistemologie studiert nicht, wie die Dinge sind, sondern wie sie gemacht sind, und in der gleichen Weise ist die Archivarbeit kein „extractive enterprise“, sondern ein „ethnographisches Unterfangen“, sagt Ann Laura Stoler (2002, S. 90) über die Arbeit in kolonialen Archiven. Eine Ethnographie der archivarischen Arbeit fragt danach, welche Vorstellungen und welches Wissen Menschen darüber entwickeln, was sie wissen und nach welchen Regeln Institutionen Wissen als Wissen anerkennen. Wer ist legitimiert, Wissen zu produzieren und wer ist in der Lage, die Prozesse der Produktion von Wissen und deren Legitimation zu verändern? Davon hängt ab, welches Wissen archiviert wird und unter welchen Bedingungen eine Sammlung von Informationen zu einem Archiv wird.

Was bedeutet vor diesem Hintergrund die Einführung des ,Anderen“ in den erziehungswissenschaftlichen Diskurs? Es handelt sich um einen epistemischen Bruch, das heißt um einen Veränderung des Realismus zweiter Ordnung. Der ,Andere“ repräsentiert aus der Perspektive der Historischen Epistemologie nicht einfach eine Realität, auch wenn er zu dieser einen losen Bezug unterhält. Der ,Andere` ist, formal betrachtet, eine Intervention in einem theoretischen, philosophischen oder wissenschaftlichen Feld.

\section{6}

Kritik ist eine treibende Kraft der politischen, ökonomischen, kulturellen und epistemischen Gewalt der europäische Moderne. Sie bleibt den Traditionen des Fortschritts, der Aufklärung und Zivilisierung, des Humanismus und seinen modifizierenden Formen des 
Antihumanismus, Posthumanismus und Transhumanismus sowie dem (neuzeitlichen) Subjekt und der Vernunft verhaftet. Diese Form der - pädagogisierenden - Kritik ist ebenso notwendig wie begrenzt. Ihre Schwäche besteht darin, dass sie ihre eigenen Grenzen nur in der Form einer excercice de la pensée anerkennt und daraus eine Stärke macht: Grenzenlose Kritik wird zum Signum der Unbegrenztheit des Denkens.

$\mathrm{Zu}$ Michael Wimmers Sprache gehören Begriffe, die einen epistemischen Bruch mit der Kritik der Moderne artikulieren, ohne sie zu verwerfen. Mit diesen Begriffen lässt sich ein Diskurs nur noch unter Vorbehalt fortsetzen. Wimmer bezieht sich auf ihn in einer Absetzbewegung oder dekonstruktiven Intervention. Aporien, Widersprüche und Paradoxien bilden die Attraktionszentren seines Denkens.

„Lässt sich also etwas gegen die reduktionistischen Bildungs- und Zukunftsvorstellungen vorbringen, das sich nicht nur einer anderen Bewertung oder Wertsetzung verdankt und nicht den Charakter eines bloß utopischen Wunschbildes oder eines Ideals hat? Ist es vielleicht möglich, eine Grenze der Verfügbarkeit über Bildungsprozesse anzugeben, etwas der ökonomischen Denkform Unzugängliches, was diese zwar als Gegeben oder sich vollziehend voraussetzen muss, ohne es aber selbst bedenken und sehen zu können? Ist die Dynamik gegenwärtiger Gesellschaften vielleicht so beschaffen, dass in ihnen selbst widerstreitende Elemente wirksam sind, die jede klare Zukunftsplanung fragwürdig werden lassen“ (S. 51)?

Jedes Denken verfängt sich in den Denkvoraussetzungen, die es hinter sich lassen will, und jede reflexive Schleife produziert neue Widerstände. Die Fragen verbieten Antworten, der Zweck des Fragens ist die Demontage des Gegenstands und die Öffnung eines totalisierenden Denkhorizonts. Pädagogik lässt sich in der Folge nur noch als Wissenschaft des Unmöglichen bestimmen. Ihr Anziehungspunkt ist das Nichtverfügbare. Randgänge dekonstruieren Vorstellungen eines Zentrums, einer Einheit und eines Allgemeinen im Denken oder in der Wissensproduktion. Der Begriff der „Ruine“ veranschaulicht das gebrochene Verhältnis zur Geschichte des pädagogischen Denkens (Wimmer, 2002):

„Diese Fragen kann ich natürlich nicht beantworten. Sie sollen nur die Perspektive deutlich machen, unter der man den gegenwärtigen Diskurs über die Zukunft der Bildung betrachten kann, wenn man nicht die Hoffnung aufgeben will, Einspruch erheben zu können, obwohl das Gebäude der klassischen Bildungstheorien, von wo aus die Kritik ihre Einwände formulierte, nur noch eine Ruine ist“ (ebd.).

„Metaphernruinen“ (ebd.) stellen dar, „was die schöne Totalität von innen zersetzt und ruiniert“ (ebd., S. 52). Sie geben den Blick auf etwas anderes frei: Alterität, der Andere und der Fremde sind die zentralen Begriffe im Werk von Michael Wimmer.

\section{7}

Der Andere bündelt die Kritik der Moderne und seiner erziehungswissenschaftlichen Reflexion: „die Relation zum - singulären, sozialen, kulturellen, geschlechtlichen, leiblichen - Anderen, die ein anderes Denken verlangt“ (Wimmer, 2002, S. 114). Der Begriff des Anderen ist zentral, weil er im Ausgang aus der europäischen Moderne eine Art Scharnierfunktion erfüllt. Es handelt sich um einen Begriff, der zwei Formen des Denkens mit verschiedenen Horizonten beweglich verbindet. Das ursprünglich französische Wort charnière stammt vom lateinischen Wort cardo und bezeichnet die Türangel, aber das spätlateinische Wort cardinalis bedeutet darüber hinaus ,wichtig‘ und ,vorzüglich“ im Sinn eines Haupt-, Angel- oder Wendepunktes. Der Begriff des Anderen bildet die Gelenkstelle zu einem anderen Denken. Er produziert und bezeichnet einen Einschnitt im Denken und einen Wendepunkt. Der Andere, eine Erfindung der europäischen Moderne, markiert die Grenze 
ihres Denkens, ihrer Kritik und Dekonstruktion. Mit dem Anderen öffnet Michael Wimmer der Erziehungswissenschaft den Ausgang zu einem anderen Denken.

\section{8}

Das Verhältnis zum Anderen begreift Wimmer im Anschluss an Castoriadis als Verhältnis zwischen Wissen und Subjekt. Eine Selbstüberschreitung der Vernunft müsse unser Verhältnis zum Wissen und zum Subjekt des Wissens verändern. Aus der Perspektive des Anderen müsse das Wissen eher als „Nichtwissen“ oder als „Nochnichtwissen“ (ebd., S. 12) gedacht werden. Wie lässt sich eine Geschichte des Anderen denken, eine Geschichte, die weder eine Entdeckungsgeschichte noch eine moderne Fortschrittsgeschichte ist und die sich nicht mehr auf den Erfahrungsraum und Erwartungshorizont der Moderne beruft (vgl. ebd., S. 13)?

\section{9}

Der/die/das Andere - die Überdeterminiertheit des Genus, nämlich die Identifikation eines geschlechtlichen Anderen mit einer strukturellen Position in der symbolischen Ordnung, signifiziert das ganze Problem mit dem Begriff des Anderen - stellt eine Konstruktion der Moderne dar, genauer, eine Konstruktion der Wahrnehmung der Krisenhaftigkeit der Moderne, aber eine Konstruktion, die nur in im Horizont der europäischen Moderne gedacht werden kann. Die kritischen Fragen verweisen nicht auf den Anderen, sondern auf „das Problematische am Problem des Anderen“ (Wimmer, 1988, S. 20). Es liege

„im Wissen selbst, das sich als Wissen über und vom Anderen präsentiert. Will man also die Nicht-Angemessenheit des Wissens nicht einfach übernehmen, dann muss zunächst danach gefragt werden, wie das Wissen strukturiert ist und durch welche Operationen es den Anderen in einer solchen Daseinsweise konstituiert und erfasst, die es dem Wissen erlaubt, sich als Wissen-von und Wissen-über zu bilden“ (ebd.).

Die Thematisierung des Anderen impliziert eine Unangemessenheit, eine Verfehlung und eine Entstellung. Nur da, wo der Andere anders bleibt, nicht verfügbar, ist der Andere anders in der Logik der (post-)modernen Konstruktion. Unverfügbarkeit bedeutet nicht Unidentifizierbarkeit. Das Andere wohnt dem Denken selbst inne und wird von diesem Denken als das hervorgebracht, was ihm entgeht.

„Nun wird auch verständlich, warum der Andere nicht als das Nicht-Identische und das Unidentifizierbare verstanden wird, als das dem Begriff Fremde oder das Undenkbare, das dem Denken äußerlich wäre. Er ist vielmehr ein Schatten, der dem Denken selbst innewohnt, ein Unwissen, das im Wissen auftaucht und stets auf es bezogen ist. Das Andere ist nicht das Andere des Wissens, das eben real und nicht Wissen ist, sondern ein potentielles Wissen vom Anderen, das noch hervorgebracht werden kann“ (Wimmer, 1988, S. 206).

Um im Werk von Wimmer eine theoretische Positionierung des Anderen an der Wende zu einem anderen Denken zu bestimmen, ist der Bezug auf die folgende Schlüsselstelle über das Verhältnis des Anderen zum Unendlichen aufschlussreich:

„Nicht mehr der Andere ist das Unendliche, sondern der Versuch, die Spaltung in zwei sich spiegelnde Unendlichkeiten aufzulösen, ist ein unendliches Unterfangen. Der Versuch, die endgültige Wahrheit zu sagen, oder bescheidener, das Wissen soweit zu vervollkommnen, dass es an einem zukünftigen Punkt aus dieser Dualität und Verdoppelung ausbrechen kann, ist die Triebfeder eines Denkens, das den Anderen 
nicht mehr als anderen anerkennen kann, sondern das Undenkbare oder das Unendliche in eine unendliche Reihe endlicher Gestalten des Anderen auflöst““(ebd.).

Wimmer weist für den Begriff des Anderen zurück, was Hegel in der Enzyklopädie der philosophischen Wissenschaften schlechte oder negative Unendlichkeit nennt: „Etwas wird ein Anderes, aber das Andere ist selbst ein Etwas, also wird es gleichfalls ein Anderes, und so fort ins Unendliche“ (Bd. 8, § 93). Daraus entsteht ein „Progreß ins Unendliche“ (Bd. 8, § 94), und es hat den Anschein, als ob die Fortsetzung der Reflexion des Anderen ad infinitum das Denken zu einem Höchsten treibe, zu einem vollkommenen Wissen, das auch noch sein ,Anderes‘ einschließe. Hegel sieht darin nur eine langweilige, oberflächliche Abwechslung: „Die Philosophie treibt sich nicht mit solchem Leeren und bloß Jenseitigen herum. Das, womit die Philosophie es zu tun hat, ist immer ein Konkretes und schlechthin Gegenwärtiges“ (§ 94).

\section{0}

In der Historischen Anthropologie ermögliche die Feststellung der Endlichkeit des Menschen, den empirischen Anderen zu entdecken. Es gehe jetzt um den konkreten Anderen, nicht mehr um den ganz Anderen oder den unendlichen Anderen. Mit der Frage nach dem Menschen ist die Absicht verbunden, auf das Dilemma zu reagieren,

„dass die Selbstermächtigung des Subjekts Voraussetzung für die Entdeckung des Anderen war und zu seiner Unterwerfung und neuerlichen Verkennung führte, wurde von der Vernunftkritik aufgedeckt, ohne von ihr aufgelöst werden zu können. Um die in der Entdeckung selbst liegende Verschlossenheit gegenüber dem Anderen öffnen zu können, ohne in eine Restauration des Heiligen und des Mythischen als einer archaischen Logik des Opfers zurückzufallen, die das Subjekt wieder einer anonymen Macht auslieferte, genügt es nicht allein, die Aufklärung zu radikalisieren oder sie über sich selbst aufzuklären. Stattdessen wäre es vielleicht aufschlussreicher, dem Verbleiben dessen nachzuforschen, wovon sich die vernünftig werdenden Subjekte emanzipieren und das sie zugleich beerben wollten: der Macht des Heiligen und der Autorität des Ganz Anderen (Wimmer, 1988, S. 282).

Statt diesem Weg zu folgen, den die Historische Anthropologie eingeschlagen hat, wird hier danach gefragt, ob es einen Ausgang aus einem Denken gibt, das danach strebt, das Ungedachte zu denken (mit dem Begriff über den Begriff hinaus), und das auf diese Weise die Grenzen der Souveränität und Mächtigkeit des - kritischen - Subjekts immer weiter ausdehnt. Ein möglicher Ausgang aus dem Denken der Moderne müsste die These des Projekts modernidad/colonialidad aufnehmen, dass die europäische Moderne und die koloniale Differenz eine Geschichte bilden und den gemeinsamen epistemologischen Rahmen für das Denken des Anderen bilden.

\section{1}

Walter Mignolo (2012) zeigt in seinem Werk, dass die Geschichte des Kapitalismus und die Geschichte der Epistemologie des Westens zusammenhängen. Das moderne Welt-System entsteht mit dem Einsetzen des Atlantic commercial circuit und fällt mit der Produktion einer kolonialen Struktur der Macht zusammen. Quijano (2007, S. 170) zufolge ist Kolonialität (coloniality) auch nach dem Ende des Kolonialismus die allgemeinste Form der Herrschaftsausübung.

„It doesn't exhaust, obviously, the conditions nor the modes of exploitation and domination between peoples. But it hasn't ceased to be, for 500 years, their main 
framework. The colonial relations of previous periods probably did not produce the same consequences, and, above all, they were not the corner stone of any global power“ (Quijano, 2007, S. 170).

\section{Die koloniale Machtstruktur erzeugt und reproduziert eine}

„specific social discrimination which later were codified as 'racial', 'ethnic', 'anthropological' or 'national', according to the times, agents, and populations involved. These intersubjective constructions, product of Euro-centered colonial domination were even assumed to be 'objective,' 'scientific,' categories, then of a historical significance. That is, as natural phenomena, not referring to the history of power“ (ebd., S. 168).

Während sich der Kolonialismus auf eine historische Periode bezieht, wird mit dem Begriff Kolonialität eine Machttechnologie bezeichnet, die heute fortbesteht und in einem ,Wissen des Anderen' gründet. Kolonialität ist nicht die Vergangenheit der Moderne, sondern ihr „anderes Gesicht“ (Castro-Gómez, 2002, S. 276).

Ein wesentliches Charakteristikum der kolonialen Machtstruktur besteht darin, dass ein imaginary, das auf race und culture gründet, einen unüberbrückbaren, hierarchisch kodierten, ausschließenden Unterschied zwischen Kolonialmächten und Kolonisierten herstellt und Kolonialisierung legitimiert. „The colonized thus appears as the ,other of reason, which justifies the use of disciplinary power by the colonizer” (ebd.). Die Produktion von Wissen, Perspektiven, Bildern und Deutungsmustern reproduziert und legitimiert politische, ökonomische und kulturelle Unterdrückung. „The epistemic colonial difference divides one from the other. Of course, this does not place one against the other but underlines the colonial difference as the limit of the assumed totality of Western epistemology. That is why to open the social sciences is a welcome move, but an insufficient one“ (Mignolo, 2002, S. 85).

\section{2}

Der Andere ist das Produkt der Moderne/der Kolonialität, wie Santiago Castro-Gómez (2002, S. 269) betont: „Modernity is an alterity-generating machine that, in the name of reason and humanism, excludes from its imaginary the hybridity, multiplicity, ambiguity, and contingency of different forms of life.“ Der Ausblick auf das Ende der Moderne/Kolonialität ist verbunden mit der Aussicht auf die Zersetzung des Anderen, den Castro-Gómez (vgl. ebd.) als Element einer binären Logik und damit als Teil einer Struktur begreift, die jede Form von Differenz (die keine Opposition hervorbringt) unterdrückt. Er spricht von der „Erfindung des Anderen“ im Kontext staatlicher Subjektivierungspolitik und versteht darunter ,the mechanisms of power/knowledge from which those representations are constructed. The problem of the ,other' must be approached theoretically not so much as the ,concealment ${ }^{\text {‘ }}$ of a preexisting cultural identity as from a perspective that takes into account the process of material and symbolic production that modern societies have been involved in since the beginning of the sixteenth century“ (ebd., S. 271f.).

Der Andere ist nur in der Imagination eines sich selbst ermächtigenden Subjekts eine Abstraktion. Was dem Denken entgeht und im Sagen nicht gesagt wird, ist nicht nur eine Frage der abstrakten Repräsentation, sondern hat eine konkrete Materialität in einem Beziehungsgefüge und ihren Institutionen wie der Schule, dem Gefängnis und Krankenhaus, dem Recht und dem Staat oder den Sozial- und Humanwissenschaften. Die systematische Verknüpfung von Wissen und Disziplinarmacht macht aus dem Projekt der Moderne für Castro-Gómez (vgl. 2002, S. 275) - er folgt den Untersuchungen von Gayatri Chakravorty Spivak - eine Praxis „epistemischer Gewalt“: „the remotely orchestrated, far-flung, and heterogeneous project to constitute the colonial subject as Other. This project is also the asymmetrical obliteration of the trace of that Other in its precarious Subject-ivity“ (Spivak, 1988, S. 280f.). 
Man kann den Anderen nicht unabhängig von der Produktion eines wissenden Subjekts denken. Es hat von sich selbst die Vorstellung einer völligen Selbsttransparenz geschaffen, „disincorporated from the known and untouched by the geo-political configuration of the world in which people are racially ranked and regions are racially configured" (Mignolo, 2009, S. 160). Nach Stephen Toulmin (vgl. 1994) hat die Geschichte der Moderne immer mit der Vorstellung kokettiert, man könne Wissen wie Kapital akkumulieren, wenn man das Wissen von seiner Geschichte befreit und bei einem Nullpunkt beginnt. Dieser fungiert als eine sich selbst durchsichtige Grundlage für eine Wissensproduktion, die nicht mehr von subjektiven Interessen kontaminiert ist.

„Bis jetzt dürfte klar sein, dass man die Hoffnung auf Gewissheit und Klarheit in der Theorie mit der Unmöglichkeit, in der Praxis Ungewissheit und Vieldeutigkeit zu vermeiden, zu einem Ausgleich bringen muss. Doch die herkömmliche Sicht der Moderne ... übernahm auch den Glauben der Rationalisten, der moderne, rationale Umgang mit Problemen bestehe darin, den ererbten Wirrwarr von Traditionen wegzufegen, reinen Tisch zu machen und wieder am Nullpunkt anzufangen“ (ebd., S. 281).

Die Einsicht in die Unmöglichkeit, ein System zu schließen und das Denken stillzustellen, verweist selbst noch auf die Hybris eines europäischen Denkens, das ihre Grenzen negiert und sich das Phantasma der Universalität eingeschrieben hat. Toulmin beschreibt die Tradition einer Wissenspolitik, die dem kolonialen Denken entspringt und in der Wissenspolitik der Europäischen Union ihren zeitgemäßen Ausdruck findet. Die Anstrengung, das Unmögliche zu denken, gehört, kritisch gewendet, der gleichen Tradition an. Demgegenüber gehe eine „geo-politics of knowledge“ mit einer "geo-politics of knowing“ einher: „Who and when, why and where is knowledge generated ...? Asking these questions means to shift the attention from the enunciated to the enunciation“ (Mignolo, 2009, S. 160). Mignolo fordert einen „epistemischer Ungehorsam“, der darin bestehe, eine Epistemologie zu entwickeln, die sich von der Illusion des Nullpunktes befreit und ,geo-historical and bio-graphical configurations“ (ebd., S. 162) in die Generierung von Wissen einführt. Auf diese Weise werden nicht nur die Inhalte, sondern die Begriffe und Regeln des Wissensdiskurses der europäischen Moderne verändert.

\section{3}

In seiner Studie Epistemologies of the South vertritt Boaventura de Sousa Santos (2014) die These, dass das moderne westliche Denken ein „abyssal thinking“ sei (wenngleich nicht die historisch einzige Form eines solchen Denkens). Santos versteht darunter ein System von sichtbaren und unsichtbaren Unterscheidungen. Die sichtbaren Unterscheidungen beruhen auf den unsichtbaren Unterscheidungen, die die soziale Realität in zwei voneinander getrennte Welten teilen. Jenseits der Grenze der akzeptierten und rechtlich legitimierten sozialen Realität gibt es eine Welt, die als nicht-existent - „nondialectical absen“ (ebd., S. 118) produziert wird und, von der sozialen Realität diesseits völlig losgelöst, nach eigenen Regeln funktioniert. Santos spricht von einer „abyssal line“, um die Grenze einer radikalen Exklusion kolonialer Gesellschaften von metropolen Gesellschaften zu bezeichnen (vgl. Sian, 2014, S. 70). Das Wort abyssal geht auf das griechische Wort ,Abyssos‘ zurück und heißt ,grundlos‘, ,unermesslich` und ,Abgrund‘. In der biblischen Mythologie taucht der Begriff ,Abyssos‘ in unterschiedlichen Zusammenhängen auf. In der Offenbarung des Johannes handelt es sich um einen Abgrund, aus dem Heuschrecken oder auch ein Drache und die Schlange aufsteigen. Bei Paulus ist Abyssos das Totenreich und bei Lukas ist es nicht nur der Ort, wo die Dämonen gefangen gehalten werden, sondern auch die Bezeichnung des trennenden Abgrunds zwischen den Gerechten und Ungerechten im Jenseits. 
Wissen und Recht sind nach Santos die beiden wichtigsten Manifestationen des ,abyssalen“ Denkens. Beide hängen miteinander zusammen, aber jeder der beiden Bereiche produziert ein eigenes System mit sichtbaren und unsichtbaren Unterschieden. Im Bereich des Wissens sind es die modernen Wissenschaften, die die Grenzen des Wissens definieren. Wissenschaftliche Kontroversen gehören zum sichtbaren Bereich, welcher das Resultat einer Grenzziehung darüber ist, was überhaupt im Feld des Wissens sichtbar werden kann und nicht davon ausgeschlossen bleibt und im besten Fall als bloße Meinung, Überzeugung, Intuition oder subjektives Alltagswissen zum Objekt von Wissenschaften wird: „I mean popular, lay, plebeian, peasant, or indigenous knowledges on the other side of the line“ (Santos, 2014, S. 119). Im Bereich des Rechts definiert die Unterscheidung zwischen legal und illegal den sichtbaren Bereich, während darunter oder daneben einer unsichtbarer Bereich existiert, in dem diese Unterscheidung kein regulierendes Prinzip darstellt. In diesem Bereich, der als vorgesellschaftlicher Naturzustand beschrieben wird, haben gesellschaftliche Institutionen keinen Platz: „The colonial is thus the blind spot upon which the modern conceptions of knowledge and law are built“" (ebd., S. 122).

\section{4}

Das Spannungsverhältnis zwischen (staatlicher) Regulation und Emanzipation definiert den sichtbaren Wissensbereich und das Feld der modernen Wissenschaften. Jenseits davon formieren Inbesitznahme, Aneignung und Gewalt eine unsichtbare Parallelgesellschaft.

Auch nach der historischen Periode des Kolonialismus hat sich das abyssale Denken nicht verändert und zieht eine Trennlinie „between the human and the subhuman in such a way that human principles do not get compromised by inhuman practices“ (ebd., S. 124). Nach einer Phase antikolonialer Kämpfe, in der für eine kurze Zeit die Hoffnung geschürt wurde, dass die abyssal line auf der globalen Ebene verschwinden könnte, hat sich in den 1970er und 1980er Jahren die Entwicklung umgekehrt: „The logic of appropriation/violence has been gaining strength to the detriment of the logic of regulation/emancipation, to such an extent that the domain of regulation/emancipation is not only shrinking but becoming internally contaminated by the logic of appropriation/violence“ (Santos, 2014, S. 125). Die Rückkehr des Kolonialen mit einer Gegenbewegung, die Santos (ebd.) „subaltern cosmopolitanism“ nennt, ist hier eine Metapher „for those who perceive their life experiences as taking place on the other side of the line and rebel against it” (ebd.).

Die neue Entwicklung eines „abyssal colonial“ vollzieht sich nicht entlang der Grenze des globalen Südens und Nordens, sie hält sich nicht an die territorialen Grenzen von Nationalstaaten, sie manifestiert sich vielmehr mitten in metropolen Gesellschaften, vorzugsweise dort, wo sich der Staat zurückzieht und soziale Regelungen des Zusammenlebens privatisiert werden. Besitznahme und Gewalt werden zum regulierenden Prinzip einer gesellschaftlichen Ordnung, die für Santos (2014, S. 128-133; vgl. auch 2002, S. 447-458) den Aufstieg des gesellschaftlichen Faschismus kennzeichnet.

Wer ist der/die/das Andere in diesem abyssalen Denken, dessen sichtbare und unsichtbare Grenzen den Globus, aber auch metropole Gesellschaften teilen? Die Dekonstruktion befasst sich mit einer spezifischen Figur des Anderen. Sie ist immer im Diesseits der Sichtbarkeit situiert. Als dasjenige, was im Denken diesem entgeht, partizipiert es am philosophischen Diskurs als sein Anderes. Mit der Kritik teilt die Dekonstruktion, dass sie zum sichtbaren Bereich gehört. Das gilt für die Dekonstruktion auch dann, wenn sie die nicht artikulierten Grundlagen des Denkens freilegt. Die Aktualisierung von Potentialitäten ist etwas anderes als die Distinktion zwischen Sichtbarkeit und Unsichtbarkeit. Der Bereich der Sichtbarkeit umfasst Aktualisierungen und Potentialitäten.

Von dieser ersten Figur und dem ersten Begriff des Anderen müsste man den ,abyssalen Anderen“ unterscheiden, den „Subhumanen“, um in der Sprache von Santos zu bleiben. 
Jenseits der abyssal line etablieren sich neue Relationen zwischen dem Subjekt und dem Anderen, aber sie lassen sich nicht in die Struktur des sichtbaren Feldes übersetzen. Can the Subaltern speak von Spivak ist eine Auseinandersetzung über die Stellung und Repräsentation des Anderen im intellektuellen Diskurs, und Der Andere und die Sprache von Wimmer ist ein Werk der Dekonstruktion und über sie, aber es bewahrt, ohne dieser Frage systematisches Gewicht zu geben, die Heterogenität des spezifischen Anderen.

\section{5}

Immanuel Wallerstein (vgl. 2000) hat gezeigt, wie die Sozialwissenschaften in die Produktion und Reproduktion des Weltsystems und der kolonialen Machtstruktur involviert sind, aber dass selbst kritische Studien diesen systematischen Zusammenhang ausblenden. Darauf weist Castro-Gómez (2002, S. 275) hin:

„The social sciences' persistent negation of this link between modernity and colonialism has been one of the clearest signs of their conceptual limitations. Permeated from the beginning with a European imaginary, the social sciences projected the idea of an aseptic and self-generating Europe, historically formed without any contact with other cultures ... From this perspective, the experience of colonialism seems to be completely irrelevant to an understanding of the phenomenon of modernity and the rise of the social sciences."

Die Sozialwissenschaften haben niemals einen epistemologischen Bruch vollzogen, sondern das abyssale Denken reproduziert. Für Castro-Gómez (ebd., S. 282) besteht die Herausforderung der Sozialwissenschaften darin, „how to name totality (with its persistent colonial face) without falling into the essentialism and universalism of metanarratives. The task of a critical theory of society is, then, to make visible the new mechanisms of colonial production of differences in times of globalization.“ Der Begriff der Totalität ist das theoretische Gegengift zur Differenzproduktion des kapitalistischen Welt-Systems. CastroGómez vermeidet damit die Verdoppelung des kapitalistischen Realismus auf der Ebene der Sozialwissenschaften.

\section{6}

Erziehungswissenschaft, die in ihre Forschungen systematisch die Voraussetzungen ihrer Wissensproduktion im Kontext der europäischen Moderne $=$ Kolonialität einbezieht, müsste heute zu einem theoretischen Konzept von Totalität zurückkehren, um kapitalistische Differenzproduktionen analysieren zu können.

\section{Nachtrag: Über den Status und die Aufgabe der Allgemeine Erziehungswissenschaft}

Michael Wimmer interveniert mit zahlreichen Beiträgen in die Entwicklung der Allgemeinen Erziehungswissenschaft. Stellvertretend zählen dazu seine Auseinandersetzung mit Wilhelm Flitners Allgemeiner Pädagogik (vgl. Wimmer, 2015), das Buch Dekonstruktion und Erziehung (Wimmer, 2006) und der Artikel Pädagogik als Kulturwissenschaft. Programmatische Überlegungen zum Status der Allgemeinen Erziehungswissenschaft (Wimmer, 2002). Die folgenden Notizen über den Status und die Aufgabe der Allgemeinen Erziehungswissenschaft sind in der Auseinandersetzung mit diesen Arbeiten und der aktuellen Entwicklung der Erziehungswissenschaft entstanden. Sie weisen einen systematischen Bezug zum Denken der Kolonialen Differenz und der Historischen Epistemologie auf, auch wenn dies nicht an jeder Stelle expliziert ist. 
(a) Allgemeine Erziehungswissenschaft ist nach meiner Auffassung keine erziehungswissenschaftliche Teildisziplin; sie verfügt über keinen von anderen Teildisziplinen empirisch abgrenzbaren Feldes (wie Schulpädagogik, Erwachsenenbildung oder Kindheitsforschung). Allgemeine Erziehungswissenschaft ist auch keine Diskurspolizei und Disziplinarmacht der Erziehungswissenschaft. Weder bestimmt sie den Kern oder die Essenz des Pädagogischen noch definiert sie allgemeinverbindlich pädagogische Grundbegriffe oder formuliert metatheoretische Grundregeln für disziplinäre Zugehörigkeit. Allgemeine Erziehungswissenschaft organisiert und reproduziert keine wissenschaftliche Arbeitsteilung (zum Beispiel zwischen Theorie und Empirie); sie ist nicht der kritische Stachel der Disziplin. Ihr Geschäft ist weder die normative Grundlegung noch die Repräsentation der Disziplin oder das Schreiben pädagogischer Hagiographien.

(b) Gegenüber solchen Positionen vertrete ich die Auffassung, dass die Allgemeine Erziehungswissenschaft eine Intervention auf dem Feld der Erziehungswissenschaft darstellt, die alle Teildisziplinen einschließt. Allgemeine Erziehungswissenschaft definiert sich durch ihre Praktiken. Ihre Intervention zielt auf eine Kritik der Wissensproduktion in einem umfassenden Sinn: Was gilt in einer bestimmten historischen Periode als pädagogisches Wissen? Wie verändert sich die Auffassung über pädagogisches und erziehungswissenschaftliches Wissen im Laufe der Zeit? Auf welche Weise wird eine pädagogische Realität zum Gegenstand eines erziehungswissenschaftlichen Wissens? Wie wird dieses Wissen legitimiert und auf welche Weise bilden sich Wissensproduktionen in der Entwicklung der Disziplin Erziehungswissenschaft ab?

(c) Eine vorläufige Definition lautet: Gegenstand der Allgemeinen Erziehungswissenschaft ist die historische und systematische Analyse von pädagogischen und erziehungswissenschaftlichen Wissensproduktionen. Diese Definition benötigt einige Erläuterungen: Erstens weise ich mit der Differenzierung zwischen pädagogischen und erziehungswissenschaftlichen Wissensproduktionen darauf hin, dass es keinen vorab definierten Wissensbegriff gibt, auf den sich Allgemeine Erziehungswissenschaft bezieht. Was unter Wissen in diesem Feld verstanden wird, ist selbst Gegenstand der Analyse. Der Begriff des Wissens definiert kein Set von bewährten Überzeugungen, er bestimmt sich durch den grauen Horizont einer ständigen Problematisierung um ein imaginary von Wissen. Diese Problematisierung artikuliert sich durch wissenschaftliche Praktiken, die ein spezifisches Wissen und damit Maßstäbe, was Wissen ist, erzeugen. Zweitens setzt Allgemeine Erziehungswissenschaft keine Definition der Disziplin voraus; Forschungen über die historische Formierung der Disziplin sind vielmehr Gegenstand der Allgemeinen Erziehungswissenschaft. Dies schließt, drittens, ein, dass Allgemeine Erziehungswissenschaft das Verhältnis der Erziehungswissenschaft zu anderen Disziplinen und transdisziplinären Forschungen untersucht und ihre Positionierung im Feld der Wissenschaft befragt.

(d) Allgemeine Erziehungswissenschaft verfolgt mit diesen Forschungen kein normatives Programm für eine Taxonomie erziehungswissenschaftlichen Wissens und keine ReFundierung der Disziplin, sondern eine systematische Reflexion der geopolitischen Dimension des Wissens und der Geschichte der kolonialen Differenz. Wenn der locus of enunciation für die Generierung von Wissen zentral ist, dann müsste jede Form der Wissensproduktion ihre eigene Begrenztheit, ihre lokale, regionale, nationale und globale Wirksamkeit zum Gegenstand der Analyse machen und dabei den Einfluss kolonialer Differenz berücksichtigen, der die Geschichte zentraler imaginaries der europäischen Moderne, die den Referenzrahmen für das pädagogische Denken und die erziehungswissenschaftliche Forschung bilden, kontaminiert.

(e) Vor dem Hintergrund einer diversality des Wissens (vgl. Mignolo, 2000) mag die folgende These auf den größten Vorbehalt stoßen: Allgemeine Erziehungswissenschaft operiert mit einem Begriff von Totalität. Gegenüber Begriffen wie Differenz, Hybridität oder Diversität erscheint der Begriff Totalität anachronistisch, und Michael Wimmer bewahrt 
gegenüber dem Totalitätsbegriff eine kritische Distanz. Es erstaunt nur auf den ersten Blick, dass ausgerechnet Castro-Gómez (2002) von den Sozialwissenschaften eine Rückbesinnung auf den Begriff der Totalität mit seinem „hartnäckigen kolonialen Gesicht“ einfordert. Ohne zu einem Essentialismus oder Universalismus eines Metanarrativs zurückzukehren, sollte „eine kritische Gesellschaftstheorie die neuen Mechanismen der kolonialen Produktion von Differenzen im Zeitalter der Globalisierung sichtbar machen“ (ebd., S. 282). Wenn der Kapitalismus in der - wissenschaftlichen - Produktion von Differenzen seine aktuelle Dynamik entfaltet, dann müsste sich eine kritische Gesellschaftstheorie zu dieser Differenzproduktion situieren und sie nicht in der Theorie verdoppeln.

(f) Ich schlage vor, die Praktiken der Allgemeinen Erziehungswissenschaft als totalisierende Operationen $\mathrm{zu}$ begreifen. Ich verstehe darunter wissenschaftliche Untersuchungen, deren Fragen und theoretische Perspektiven so angelegt sind, dass sie ,alles ‘ affizieren. Damit soll gesagt sein, dass eine theoretische Operation in ihrer Wirkung, die sie entfaltet, keine ,natürliche‘ Grenze kennt, und zweitens, dass sich eine Totalität nur in einer Praktik realisiert und nicht vor ihr existiert. Der Begriff ,alles' ist ein schwacher Hilfsbegriff, ein ungeschickter Platzhalter, denn mit jeder Realisierung dehnt sich der Horizont der Totalität aus, so dass wir von Totalität weder ein Konzept noch eine Vorstellung haben und ein solches Konzept oder eine solche Vorstellung für die wissenschaftliche Arbeit auch nicht benötigen. Totalität verkehrt sich nach diesem Verständnis in das Gegenteil der Auffassung, die man dem Konzept unterstellt hat. Totalität bewahrt vor Schließungen, indem sie jede Verkettungsform nicht nur für zulässig hält; totalisierende Operationen experimentieren mit neuen Verkettungen - der Begriff articulation in den Cultural Studies besagt genau dies. Relationen produzieren Elemente und nicht umgekehrt. Die Relationalität bewahrt die Analyse vor Universalismen, denn Relationalität bedeutet die Beachtung des locus of enunciation. Eine totalisierende Operation wäre Bachelard zufolge die „Realisierung“ eines wissenschaftlichen Gegenstandes. Marcel Mauss` Begriff einer fait social total - Mauss hat anstelle des Wortes ,total` zunächst das Wort ,allgemein` verwendet - würde einer solchen totalisierenden Realisierung entsprechen (vgl. Mauss, 1973).

(f) Die strategische Funktion der Allgemeinen Erziehungswissenschaft besteht darin, dass ihre (historischen) Analysen von Wissensproduktionen die metaphysischen Grundlagen von Forschungen, nämlich das Phantasma eines fingierten Nullpunktes in der Wissensproduktion und die Vorstellung, dass erziehungswissenschaftliches Wissen wie Kapital akkumuliert werden kann, einer Kritik unterzieht (vgl. Forster, 2016).

(g) Wenn man unter diesen Gesichtspunkten das Buch Der Andere und die Sprache interpretiert, dann lässt sich das Konzept der Totalität ebenso erkennen, wie die Kritik eines erziehungswissenschaftlichen Realismus zweiter Ordnung: Die Einführung des ,Anderen“ affiziert die gesamte Pädagogik. Die Struktur des Werks und der Modus des Fragens repräsentieren diese totalisierende Operation. Es wäre aber völlig verfehlt, den ,Anderen ‘ als Zentrum eines neuen Denkens zu begreifen, von dem aus eine Struktur seine Bedeutung erhält. Die Einführung des Anderen ist nichts anderes als die ,Realisierung، eines erziehungswissenschaftlichen Objekts. In dieser Realisierung markiert der Andere einen epistem(olog)ischen Bruch. Der Andere ist nicht selbst Objekt, sondern er ist der Modus der Realisierung eines jeden Objekts als wissenschaftliche Objekt und er repräsentiert zugleich das Gedächtnis der Realisierung, des Umstands also, dass diese Objekte wissenschaftlich hergestellt sind. Man könnte dies den dekonstruktiven Zug in der Operation der Realisierung nennen. Der Andere und die Sprache ist ein überbordendes Buch. Totalisierende Operationen sind Aktualisierungen einer virtuellen Totalität. „Das Wirkliche ist von einer unbestimmten Zone nicht-verwirklichter Möglichkeiten umgeben; die Wahrheit ist nicht der erhabenste Wert der Erkenntnis“ (Veyne, 1988, S. 42; vgl. auch Veyne, 1992). Allgemeine Erziehungswissenschaft ist eine Intervention im Feld der Erziehungswissenschaft, um ihre Wissensproduktion zu verändern. Wimmer (2002, S. 119) geht es um „die Dekonstruktion der 
Verwicklung der Pädagogik mit denjenigen Verhältnissen, die heute ihren Horizont zu verschließen drohen, und zugleich die Erneuerung der Idee einer kritischen Erziehungswissenschaft, die gegen diese fatalen Strategien Widerstand entwickeln konnte“.

\section{Literatur}

Bachelard, Gaston, Der neue wissenschaftliche Geist, Frankfurt am Main, 1988.

Castro-Gómez, Santiago, The social sciences, epistemic violence, and the Problem of the „Invention of the Other“, in: Nepantla: Views from the South, 3. Jg., 2002, H. 2, S. 269285.

Chakrabarty, Dipesh, Provincializing Europe: Postcolonial thought and historical difference, Princeton, 2008.

Derrida, Jacques, Positionen - Gespräche mit Henri Ronse, Julia Kristeva, Jean-Louis Houdebine, Guy Scarpetta, Graz und Wien, 1986.

Escobar, Arturo, Worlds and knowledges otherwise, in: Cultural Studies, Jg. 21, 2007, S. 179-210.

Forster, Edgar, Europäische Wissenspolitik der Bildung, in: Bildung und Teilhabe, hg. von Ingrid Miethe, Anja Tervooren und Norbert Ricken, Wiesbaden, 2016, im Druck.

Hegel, Georg Wilhelm Friedrich, Werke in 20 Bänden, Band 8-10: Enzyklopädie der philosophischen Wissenschaften, Frankfurt am Main, 1986.

Lorde, Audre, „The Master's Tools Will Never Dismantle the Master's House“, in: Sister Outsider: Essays and Speeches, Berkeley, 1984, S. 110-114.

Mauss, Marcel, Essai sur le don: Forme et raison de l'échange dans les sociétées archaïques, in: Sociologie et Anthropologie, Paris, 1973, S. 149-279.

Mignolo, Walter D., The many faces of cosmopolis: Border thinking and critical cosmopolitanism, in: Public Culture, 12, 2000, S. 721-748.

-, The Geopolitics of Knowledge and the Colonial Difference, in: The South Atlantic Quarterly, Jg. 101, H. 1, 2002, S. 57-96.

-, Epistemic Disobedience, Independent Thought and Decolonial Freedom, in: Theory, Culture \& Society, Jg. 26, H. 7-8, 2009, S. 159-181.

-, Local histories/global designs: Coloniality, subaltern knowledges, and border thinking, Princeton, 2012.

Quijano, Aníbal, „Coloniality and modernity/rationality“, in: Cultural Studies, Jg. 21, 2007, S. 168-178.

Rheinberger, Hans-Jörg, Historische Epistemologie zur Einführung, Hamburg, 2007.

Santos, Boaventura de Sousa, Toward a New Legal Common Sense : Law, Globalization, and Emancipation, London, 2002.

-, Epistemologies of the South. Justice against Epistemicide, Boulder und London, 2014.

Sian, Katy P. (Hrsg.), Conversations in Postcolonial Thought, New York, 2014.

Spivak, Gayatri C., Can the Subaltern speak? In: Marxism and the Interpretation of Culture, hg. von Cary Nelson und Lawrence Grossberg, Urbana und Chicago, 1988, S. 271-313.

Stoler, Ann Laura, Colonial archives and the arts of governance, in: Archival Science, Jg. 2, 2002, S. 87-109.

Toulmin, Stephen, Kosmopolis. Die unerkannten Aufgaben der Moderne, Frankfurt am Main, 1994.

Veyne, Paul, Die Originalität des Unbekannten. Für eine andere Geschichtsschreibung, Frankfurt am Main, 1988.

-, Foucault: Die Revolutionierung der Geschichte, Frankfurt am Main, 1992.

Wallerstein, Immanuel, Open the Social Sciences, in: Asia-Pacific Social Sciences Review, Jg. 1, 2000, H. 1, S. 1-10. 
Wimmer, Klaus-Michael, Der Andere und die Sprache. Vernunftkritik und Verantwortung, Berlin, 1988.

Wimmer, Michael, „Pädagogik als Kulturwissenschaft. Programmatische Überlegungen zum Status der Allgemeinen Erziehungswissenschaft“, in: Forschungsfelder der Allgemeinen Erziehungswissenschaft, hg. v. Lothar Wigger, Opladen, 2002, S. 109-122.

-, Dekonstruktion und Erziehung. Studien zum Paradoxieproblem in der Pädagogik, Bielefeld, 2006.

-, „Das Imaginäre pädagogischer Grundgedanken, der Un-Fug pädagogischer Kategorien und das Gespenst des Pädagogischen“, in: Wilhelm Flitner. Jugendbewegung, Erwachsenenbildung und Erziehungswissenschaft, hg. v. Peter Faulstich, Weinheim und Basel, 2015, S. 49-78.

Wulf, Christoph/Kamper, Dietmar (Hg.), Logik und Leidenschaft - Erträge Historischer Anthropologie, Berlin, 2002. 